\title{
ESSENTIAL DIMENSION OF ALBERT ALGEBRAS
}

\author{
MARK L. MACDONALD
}

\begin{abstract}
This paper shows that the number of independent parameters required to describe an Albert algebra up to isomorphism is at most 7 . In other words, the essential dimension of the split group of type $F_{4}$ over a field of characteristic not 2 or 3 satisfies ed $\left(F_{4}\right) \leq 7$. This is achieved by reducing the structural group from the full 52-dimensional automorphism group to a subgroup of dimension 10, and exhibiting an 18-dimenional generically free linear representation which remains generically free once projectivized.
\end{abstract}

An Albert algebra is a certain type of 27-dimensional non-associative algebra over a field (see 1.2 for a definition; also [SV00] [Alb47]). If the field is algebraically closed, then there is only one Albert algebra, but for arbitrary fields there are many open questions about their classification up to isomorphism. The basic question being addressed in this paper is:

Question 0.1. How many algebraically independent parameters do we need to describe an arbitrary Albert algebra up to isomorphism?

To make this precise, let me introduce some terminology. Say we have an inclusion of fields $F \subset F^{\prime} \subset F^{\prime \prime}$. Let $A$ be an algebra over $F^{\prime \prime}$, and let $B$ be an algebra over a subfield $F^{\prime}$ such that $A \cong B \otimes_{F^{\prime}} F^{\prime \prime}$ (isomorphic as algebras over $F^{\prime \prime}$ ). Then we say $F^{\prime}$ is a field of definition of $A$. In this situation, $A$ is an Albert algebra if and only if $B$ is. So, with respect to Question 0.1, at most tr. $\operatorname{deg}_{F} F^{\prime}$ independent parameters are needed to describe $A$.

Fix a base field $F$. Consider the smallest number $d$ such that for any Albert algebra over any field extension of $F$ there exists a field of definition whose transcendence degree over $F$ is at most $d$. This number, which is known to be finite, may be taken as an answer to Question 0.1. In this paper we show that it is at most 7 (see Theorem $0.3)$.

0.2. Essential dimension. It is known that isomorphism classes of Albert algebras correspond to isomorphism classes of $F_{4}$-torsors, for the split exceptional group of type $F_{4}$ [KMRT, 37.11]. The essential dimension of an algebraic group $G$ is a non-negative integer which has the property that $\operatorname{ed}(G)=0$ precisely for the special groups, which were classified by Grothendieck (for a definition, see Section 1). So one could think of $\operatorname{ed}(G)$ as measuring how complicated $G$-torsors can be, up to isomorphism. In fact, the above number $d$ (and hence our answer to Question 0.1) is equal to ed $\left(F_{4}\right)$.

It is known that $5 \leq \operatorname{ed}\left(F_{4}\right)$ due to the existence and non-triviality of Serre's degree 5 cohomological invariant of Albert algebras [GMS, Thm. 22.5] [BF03, Cor. 3.6]. Previously, the best known upper bound was $\operatorname{ed}\left(F_{4}\right) \leq 19$ for fields of characteristic zero [Le04]. The main result of the present paper is the following theorem.

Theorem 0.3. If the base field is of characteristic not 2 or 3, then $\operatorname{ed}\left(F_{4}\right) \leq 7$.

The proof is given at the end of the paper. The essential dimensions of the exceptional algebraic groups remain largely open (see [Re10] or [Me13] for recent surveys). For

\footnotetext{
Key words and phrases. Essential dimension, Albert algebras, generically free representations.
} 
example, even if the base field is the complex numbers, we only know that $9 \leq \operatorname{ed}\left(E_{8}\right) \leq$ 231.

Corollary 0.4. If the base field is of characteristic not 2 or 3, then $\operatorname{ed}\left(E_{6}^{s . c}\right) \leq 8$.

Proof. We know ed $\left(E_{6}^{\text {s.c. }}\right) \leq \operatorname{ed}\left(F_{4}\right)+1$ by $[\operatorname{Re} 00,11.7]$ or $[\mathrm{Ga09}, 9.12]$.

The main difficulty in the proof is showing the generic freeness of a certain representation (Theorem 3.1). Showing that the stabilizer of a point in general position of this representation is finite is not hard, since this can be done on the level of Lie algebras. But to show it is trivial requires careful consideration of the group action.

To finish this introduction, I will mention how the values of the related essential $p$-dimensions of Albert algebras are already known for all primes $p$ (see [Me13] for a definition and recent results). We know $\operatorname{ed}\left(F_{4} ; 2\right)=5$, because any Albert algebra becomes reduced after some degree prime-to-2 extension, and an arbitrary reduced Albert algebra only requires 5 parameters to describe it, by Jacobson's coordinatization [Mac08]. Also, ed $\left(F_{4} ; 3\right)=3$, because any Albert algebra becomes a first Tits construction $J(A, \lambda)$ after some degree prime-to- 3 extension, and they require three parameters to describe them: two parameters for $A$ (the degree 3 central simple algebra, which must be cyclic), and one parameter for $\lambda$. Finally, ed $\left(F_{4} ; p\right)=0$ for all primes $p \geq 5$, because any Albert algebra splits after a degree 6 extension. As usual, the essential $p$-dimensions give lower bounds for the essential dimension, but they are otherwise, apparently, not helpful in finding ed $\left(F_{4}\right)$.

\section{Preliminaries}

Unless otherwise specified, $F$ will denote an arbitrary field of characteristic $\neq 2,3$, and $\bar{F}, F_{\text {sep }}$ its algebraic and separable closures respectively. An algebraic group $G$ will be a smooth affine group scheme of finite type over a field; in particular all algebraic groups are assumed to be linear [KMRT, 21]. By a subgroup $H \subset G$ we will mean a closed subgroup scheme (not necessarily smooth). $X(F)$ will denote the set $X(\operatorname{Spec} F)$ of $F$-rational points of $X$. For an algebraic group $G$, the $\operatorname{symbols}^{\operatorname{Norm}_{G}(H)}$ or $\operatorname{Norm}_{G}(Y)$ will denote the scheme-theoretic normalizer of $G$ which preserves a subgroup $H$ or subscheme $Y$ respectively; the normalizer subgroup need not be smooth [Ja03]. In particular, if $G$ acts on $X$ and $x \in X(F)$, then the symbol $G_{x}:=\operatorname{Norm}_{G}(x)$ will denote the subgroup in $G$ of elements fixing $x$. The notation $\left\langle\left\{x_{i}\right\}\right\rangle \subset V$ will mean the vector subspace generated by some collection of vectors $\left\{x_{i}\right\} \subset V$.

We will say that a $G$-variety $X$ is generically free if there is an open dense subscheme $U \subset X$ such that the (scheme-theoretic) stabilizer of each point in $U$ is trivial. By $[\mathrm{BF} 03,4]$ it is enough to check $G_{x}=\{1\}$ for all $x \in U(\bar{F})$. For an algebraic group $G$, we will define the essential dimension of $G$ to be the minimum value of $\operatorname{dim}(X)-\operatorname{dim}(G)$, where $X$ is a generically free $G$-variety and there exists a $G$-equivariant dominant map $V \rightarrow X$ (also called a compression), such that $V$ is a generically free linear representation of $G$. By [BF03, Cor. 6.16], this is equal to Merkurjev's definition of essential dimension in terms of the étale cohomology functor $F^{\prime} \mapsto H_{e t}^{1}\left(F^{\prime}, G\left(F_{\mathrm{sep}}^{\prime}\right)\right)$ (since $G$ is assumed to be smooth, this is the same as the functors for Galois or fppf cohomology).

To understand the representation in Section 3, we will find it convenient to view the vectors simultaneously in terms of octonions and in terms of weight spaces.

1.1. Octonions. The split octonion algebra over a field $F$ will be denoted by $\mathbb{O}$, and is defined as follows. Its elements are pairs of 2 by 2 matrices over $F$, which also defines 
its vector space structure. We will define a conjugation operation

$$
\overline{\left(\begin{array}{cc}
a_{11} & a_{12} \\
a_{21} & a_{22}
\end{array}\right)}=\left(\begin{array}{cc}
a_{22} & -a_{12} \\
-a_{21} & a_{11}
\end{array}\right),
$$

and then the multiplication on the 8-dimensional vector space is defined by

$$
(a, b)(c, d)=(a c+\bar{d} b, d a+b \bar{c}), \text { where } a, b, c, d \in M_{2}(F) .
$$

The norm on the octonions is defined as $n((a, b)):=\operatorname{det}(a)-\operatorname{det}(b)$. This presentation follows [SV00, 1.8].

We will also denote standard elements

$$
1:=\left(\left(\begin{array}{ll}
1 & 0 \\
0 & 1
\end{array}\right), 0\right), \quad i:=\left(\left(\begin{array}{cc}
1 & 0 \\
0 & -1
\end{array}\right), 0\right), \quad j:=\left(\left(\begin{array}{ll}
0 & 1 \\
1 & 0
\end{array}\right), 0\right), \quad k:=\left(0,\left(\begin{array}{ll}
1 & 0 \\
0 & 1
\end{array}\right)\right) .
$$

Then the set $\{1, i, j, i j, k, i k, j k,(i j) k\} \subset \mathbb{O}$ is an $F$-vector space basis. One checks that $i, j$, and $k$ pairwise anti-commute and square to the identity 1 .

1.2. Albert algebras. Consider the vector space $J_{s}$ of 3 by 3 matrices with entries in the split octonion algebra, which are hermitian (elementwise conjugation equals matrix transpose). As a vector space, we have

$$
J_{s}:=\left\{x \in M_{3}(\mathbb{O}) \mid \bar{x}^{t}=x\right\}=\left\{\left(\begin{array}{ccc}
\lambda_{0} & c_{2} & \overline{c_{1}} \\
\overline{c_{2}} & \overline{\lambda_{1}} & c_{0} \\
\overline{c_{1}} & \overline{c_{0}} & \lambda_{2}
\end{array}\right) \mid \lambda_{n} \in F, c_{n} \in \mathbb{O}\right\} .
$$

We will also sometimes consider elements $\left(c_{0}, c_{1}, c_{2}\right) \in J_{0} \oplus J_{1} \oplus J_{2}$. Here $J_{n}=\mathbb{O}$ as vector spaces for each $n=0,1,2$; we refer to these as the Peirce subspaces.

If we equip $J_{s}$ with the multiplication $x \circ y:=\frac{1}{2}(x y+y x)$, where concatenation is the usual matrix product in $M_{3}(\mathbb{O})$, then we call it the split Albert algebra over $F$. An algebra $J$ over a field $F$ is an Albert algebra if for some field extension $J \otimes_{F} F^{\prime}$ is isomorphic to the split Albert algebra over $F^{\prime}$.

Define the 9-dimensional and 3-dimensional subalgebras as follows

$$
E_{s}:=\left\{\left(\begin{array}{ccc}
\lambda_{0} & c_{2} & \overline{c_{1}} \\
\overline{c_{2}} & \lambda_{1} & c_{0} \\
\bar{c}_{1} & \overline{c_{0}} & \lambda_{2}
\end{array}\right) \mid \lambda_{n} \in F, c_{n} \in\langle 1, i\rangle\right\}, \quad L_{s}:=\left\{\left(\begin{array}{ccc}
\lambda_{0} & 0 & 0 \\
0 & \lambda_{1} & 0 \\
0 & 0 & \lambda_{2}
\end{array}\right) \mid \lambda_{n} \in F\right\} .
$$

Then $J_{s} \supset E_{s} \supset L_{s}$. We will say an automorphism preserves a subalgebra, if the image of the subalgebra is itself.

The algebra automorphism group $\operatorname{Aut}\left(J_{s}\right)$ is the split group of type $F_{4}$. Over an algebraically closed field there is only one Albert algebra up to isomorphism.

Remark 1.3. It is a non-trivial fact from the structure theory of Jordan algebras, that over $\bar{F}$, the Albert algebra is the only simple Jordan algebra which does not "come from" an associative algebra. While much is known about the classification of Albert algebras over non-algebraically closed fields, several open questions remain; for example, Serre asked whether Albert algebras are classified by their cohomological invariants with coefficients in $\mathbb{Z} / 2$ and $\mathbb{Z} / 3$, and this is still open [Se93, 9.4].

1.4. $\operatorname{Spin}_{8} \rtimes S_{3} \subset F_{4}$. It is well known that the group of automorphisms preserving the diagonal matrices in $J_{s}$ is $\operatorname{Norm}_{F_{4}}\left(L_{s}\right) \cong \operatorname{Spin}_{8} \rtimes S_{3}$ [KMRT, 39.13]. Here transpositions in $S_{3}$ act on $J_{s}$ by matrix transpose composed with swapping diagonal idempotents, and swapping their corresponding Peirce subspaces [Ga05, 3.2]. We will find it convenient to express the subgroup $\mathrm{Spin}_{8}$ (as a scheme) as a subgroup of $\mathrm{SO}_{8}^{3}$, defined by its action on $J_{0} \oplus J_{1} \oplus J_{2}=\mathbb{O}^{3}$. In particular, by [KMRT, 35.8]:

$$
\operatorname{Spin}_{8}(F) \cong\left\{\left(g_{0}, g_{1}, g_{2}\right) \in \mathrm{SO}_{8}^{3} \mid g_{0}(x \cdot y)=\overline{g_{2}(\bar{x})} \cdot \overline{g_{1}(\bar{y})}, \forall x, y \in \mathbb{O}\right\} .
$$


Here the multiplication is the usual one in $\mathbb{O}$. Given such a triple $\left(g_{0}, g_{1}, g_{2}\right)$, we can extend to a vector space automorphism of $J_{s}$ by taking the identity on $L_{s}$. One checks this defines an algebra automorphism of $J_{s}$. Notice that a different presentation of $\mathrm{Spin}_{8}$ was used in [Bö11], so in what follows our statements will differ slightly from his.

1.5. $\mathrm{SL}_{2} \subset \mathrm{SL}_{3} \subset G_{2} \subset \mathrm{Spin}_{8}$. The triples in $\operatorname{Spin}_{8}$ such that $g_{0}(1)=g_{1}(1)=g_{2}(1)=$ 1 are exactly the triples such that $g_{0}=g_{1}=g_{2}=g$ on $\mathbb{O}$, where $g \in \operatorname{Aut}(\mathbb{O})$ [KMRT, 35.16]. This is the split group of type $G_{2}$, and we will abuse notation slightly by referring to this subgroup in $\operatorname{Spin}_{8}$ as $G_{2}$. Similarly we will denote the subgroups $\mathrm{SL}_{3}:=\operatorname{Norm}_{G_{2}}(i)$ and $\mathrm{SL}_{2}:=\operatorname{Norm}_{\mathrm{SL}_{3}}(j)$ which are smooth subgroups of the given types [SV00, 2.2].

1.6. $T_{2} \subset \operatorname{Spin}_{8}$. For $\lambda \in F^{*}$, let $R_{\lambda}$ (resp. $L_{\lambda}$ ) be the linear isomorphism of $\mathbb{O}$ given by right (resp. left) multiplication by $\left(\left(\begin{array}{cc}\lambda & 0 \\ 0 & \lambda^{-1}\end{array}\right), 0\right)$. Then one checks (using the Moufang identities) that the following elements of $\mathrm{SO}_{8}^{3}$ live inside $\mathrm{Spin}_{8}$.

$$
r_{\lambda}:=\left(L_{\lambda}, R_{\lambda}, L_{\lambda^{-1}} \circ R_{\lambda^{-1}}\right), \quad s_{\mu}:=\left(R_{\mu}, L_{\mu^{-1}} \circ R_{\mu^{-1}}, L_{\mu}\right) .
$$

For example, if we let $\left(g_{0}, g_{1}, g_{2}\right)=s_{\lambda}$, and $x, y \in \mathbb{O}, a:=\left(\begin{array}{cc}\lambda & 0 \\ 0 & \lambda^{-1}\end{array}\right)$, then

$$
\overline{g_{2}(\bar{x})} \cdot \overline{g_{1}(\bar{y})}=(x \cdot \bar{a}) \cdot((a \cdot y) \cdot a)=(((x \cdot \bar{a}) \cdot a) \cdot y) \cdot a=(x \cdot y) \cdot a=g_{0}(x \cdot y) .
$$

Furthermore, these elements generate two one-dimensional tori which intersect in the identity and commute. The two-dimensional split torus they generate will be denoted by $T_{2} \subset \operatorname{Spin}_{8}$.

1.7. Decomposition of $J_{s}$. Consider the (split) maximal torus $T_{4} \subset F_{4}$ generated by $T_{2}$ above and the 2-dimensional torus in $\mathrm{SL}_{3}$ described in [SV00, 2.3]. Then $J_{s}$ has 24 non-zero $T_{4}$-weights, each of which has a 1-dimensional weight space and corresponds to a short root of $F_{4}$. So as an $F_{4}$ representation, $J_{s}=F \oplus V_{26}$, the sum of a trivial representation, and the 26-dimensional irreducible representation.

As in 1.1 , we can write any octonion (and therefore any element of $J_{0}, J_{1}$, or $J_{2}$ ) as a pair of 2 by 2 matrices. It turns out that the non-zero $T_{4}$-weight spaces are exactly the matrix entries from the pairs of 2 by 2 matrices in $J_{0}, J_{1}$, and $J_{2}$. Using the notation for the $F_{4}$-roots from [Bour], we can label the weight spaces as follows:

$$
\begin{gathered}
\left.J_{0}: \quad\left(\begin{array}{cc}
\epsilon_{1} & \epsilon_{2} \\
-\epsilon_{2} & -\epsilon_{1}
\end{array}\right),\left(\begin{array}{cc}
\epsilon_{3} & -\epsilon_{4} \\
\epsilon_{4} & -\epsilon_{3}
\end{array}\right)\right), \\
J_{1}: \quad\left(\left(\begin{array}{cc}
(-+++) & (-+--) \\
(+-++) & (+---)
\end{array}\right),\left(\begin{array}{ll}
(--+-) & (+++-) \\
(---+) & (++-+)
\end{array}\right),\right. \\
J_{2}: \quad\left(\left(\begin{array}{cc}
(----) & (++--) \\
(--++) & (++++)
\end{array}\right),\left(\begin{array}{ll}
(+-+-) & (-++-) \\
(+--+) & (-+-+)
\end{array}\right)\right)
\end{gathered}
$$

Here the notation $(-+++)$ means, for example, the weight $\frac{1}{2}\left(-\epsilon_{1}+\epsilon_{2}+\epsilon_{3}+\epsilon_{4}\right)$.

The roots of $\operatorname{Spin}_{8}$ are given by the 24 long roots of $F_{4}$, which are $\pm \epsilon_{m} \pm \epsilon_{n}, m<$ $n$. With this notation, the above subgroups $\mathrm{SL}_{2}$ and $\mathrm{SL}_{3}$ have roots $\pm\left(\epsilon_{3}-\epsilon_{4}\right)$ and $\pm\left(\epsilon_{m}-\epsilon_{n}\right), 2 \leq m<n \leq 4$ respectively. Furthermore, as $\mathrm{SL}_{3}$-representations, each of $J_{0}, J_{1}$, and $J_{2}$ are a direct sum of a 2-dimensional trivial representation, the standard 3 -dimensional representation, and its 3-dimensional dual.

From 1.2, $L_{s}$ is the 3 -dimensional zero weight space, and $E_{s}$ is that together with the six weight spaces from the diagonals of the three matrices on the left-hand sides, above. 


\section{Reduction to A SUbGroup}

We will denote by $F_{4}$ the split group of that type over the base field. To give the new upper bound on ed $\left(F_{4}\right)$, we will reduce to a 10-dimensional subgroup, $G \subset F_{4}$, which has more small representations than $F_{4}$ does.

2.1. The subgroup $G \subset F_{4}$. Using the notation from 1.2 , we will define $G$ to be the subgroup of automorphisms of $J_{s}$ which preserve both $E_{s}$ and $L_{s}$,

$$
G:=\operatorname{Aut}\left(J_{s}, E_{s}, L_{s}\right):=\operatorname{Norm}_{\mathrm{Norm}_{F_{4}}\left(L_{s}\right)}\left(E_{s}\right) .
$$

The notation $(J, E, L)$ will refer to a triple of algebras defined over $F$, such that and $J \supset E \supset L$. An isomorphism between two such triples is an isomorphism between the largest algebras, which also restricts to respective isomorphisms on the two subalgebras.

Definition 2.2. An algebra $A$ is a form of an algebra $B$ if there is a field extension $F^{\prime}$ such that $A \otimes F^{\prime} \cong B \otimes F^{\prime}$. A triple $(J, E, L)$ will be called a form of $\left(J_{s}, E_{s}, L_{s}\right)$, if after some base field extension, it becomes isomorphic to $\left(J_{s}, E_{s}, L_{s}\right)$.

Lemma 2.3. Assume $(J, E, L)$ is a triple such that $J, E$, and $L$ are forms of $J_{s}, E_{s}$, and $L_{s}$, respectively. Then $(J, E, L)$ is a form of $\left(J_{s}, E_{s}, L_{s}\right)$.

Proof. It is enough to assume the field is separably closed, and then prove that $(J, E, L)$ becomes isomorphic to $\left(J_{s}, E_{s}, L_{s}\right)$. Choose an isomorphism $\phi_{0}$ sending $J_{s}$ to $J$. Now there is an automorphism $\phi_{1}$ of $J$ which sends $\phi_{0}\left(E_{s}\right)$ to $E$, by [KMRT, 37.20]. Finally, there is an automorphism $\phi_{2}$ of $J$ preserving $E$ which sends $\phi_{1}\left(\phi_{0}\left(L_{s}\right)\right)$ to $L$, by coordinatization together with [KMRT, 37.20]. So $\phi_{2} \circ \phi_{1} \circ \phi_{0}$ sends the triple $\left(J_{s}, E_{s}, L_{s}\right)$ to $(J, E, L)$.

Lemma 2.4. The Galois cohomology set $H^{1}(F, G)$ is in bijective correspondence with the set of isomorphism classes of forms of $\left(J_{s}, E_{s}, L_{s}\right)$.

Proof. Using the arguments of [KMRT, 29.C], this follows from Lemma 2.3.

The following proposition was proved in [Bö11] over the complex numbers.

Proposition 2.5. $G \cong\left(\left(\left(\mathrm{SL}_{3} \times \mathbb{G}_{m}^{2}\right) / \mu_{3}\right) \rtimes \mathbb{Z} / 2\right) \rtimes S_{3}$ as group schemes.

Proof. We have $G \subset \operatorname{Norm}_{F_{4}}\left(L_{s}\right) \cong \operatorname{Spin}_{8} \rtimes S_{3}$, and let $G^{\prime}$ be the kernel of the $G$ action on $L_{s}$, so $G^{\prime} \subset \operatorname{Spin}_{8}$. Since $S_{3}$ preserves $E_{s}$, we have $G \cong G^{\prime} \rtimes S_{3}$.

The subgroup Aut $(\mathbb{O},\langle 1, i\rangle):=\operatorname{Norm}_{G_{2}}(\langle i\rangle)$ is isomorphic to $\mathrm{SL}_{3} \rtimes \mathbb{Z} / 2 \subset \operatorname{Spin}_{8}$ [Ja58, Thm. 4]. Notice that both $\mathrm{SL}_{3} \rtimes \mathbb{Z} / 2$ and $T_{2}$ are contained in $G^{\prime}$, so consider the homomorphism of group schemes $f:\left(\mathrm{SL}_{3} \rtimes \mathbb{Z} / 2\right) \times T_{2} \rightarrow G^{\prime}$ induced by the group product. $f$ is surjective, because for any $g \in G^{\prime}$, we can find $\lambda, \mu \in F^{*}$ such that $g \cdot r_{\lambda} s_{\mu}$ fixes $(1,1,1) \in J_{0} \oplus J_{1} \oplus J_{2}$. The subgroups $\mathrm{SL}_{3} \rtimes \mathbb{Z} / 2$ and $T_{2}$ commute, and the $F$-points of $\operatorname{ker}(f)$ coincide with the $F$-points of $\mu_{3}$. By [KMRT, 22.13], the result will now follow if $d f$ is a surjection of Lie algebras.

By $[\operatorname{KMRT}, 21.5(7)]$, we have $\operatorname{Lie}(G)=\left\{h \in \operatorname{Lie}\left(\operatorname{Spin}_{8}\right) \mid h\left(E_{s}\right) \subset E_{s}\right\}$. Assume $h \in \operatorname{Lie}(G)$, and write $h=h_{0}+\sum_{\alpha} h_{\alpha}$, where $\alpha$ runs over the $\operatorname{Spin}_{8}$-roots, and $h_{0} \in \operatorname{Lie}\left(T_{4}\right)$.

We claim that if $\beta$ is a $T_{4}$-weight of $E_{s}, \alpha$ a $\operatorname{Spin}_{8}$-root, and $\alpha+\beta$ is a weight of $J_{s}$, then $h_{\alpha}=0$. In this situation, if $h_{\alpha} \neq 0$, then $h_{\alpha} V_{\beta}=V_{\alpha+\beta}$, since they are both 1-dimensional weight spaces; they could also be viewed as root spaces of $\operatorname{Lie}\left(F_{4}\right)$. But this contradicts the fact that $h$ preserves $E_{s}$, since $\alpha+\beta$ cannot be a weight of $E_{s}$, proving the claim.

By considering $\beta= \pm \epsilon_{1}$, this shows that $h_{\alpha} \neq 0$ implies $\alpha= \pm \epsilon_{m} \pm \epsilon_{n}$, where $2 \leq m<n \leq 4$. By arguing similarly using $\beta= \pm(-+++)$, we see in fact $\alpha$ must be 
a root of $\mathrm{SL}_{3}$. So $\operatorname{Lie}(G) \subset \operatorname{Lie}\left(\mathrm{SL}_{3}\right) \oplus \operatorname{Lie}\left(T_{2}\right)$, and the reverse containment is obvious. This completes the proof.

Theorem 2.6. For any field $F$, the inclusion $G \subset F_{4}$ induces a surjection $H^{1}(F, G) \rightarrow$ $H^{1}\left(F, F_{4}\right)$.

Proof. The map to $H^{1}\left(F, F_{4}\right)$ is given by the forgetful functor $(J, E, L) \mapsto J$. To see surjectivity, take a pair of elements $x, y \in J$, and let $L$ be the subalgebra generated by $x$, and let $E$ be the subalgebra generated by $x$ and $y$. For generic $x$ and $y$ this creates a form of $\left(J_{s}, E_{s}, L_{s}\right)$ as required [KMRT, 39.20]. Notice when $F$ is finite, then $H^{1}\left(F, F_{4}\right)$ is trivial.

\section{Generic Freeness}

For each $n=0,1,2$, let $V_{n} \subset J_{n}$ be the 6-dimensional subspace as follows

$$
V_{n}:=\left(\left(\begin{array}{cc}
0 & * \\
* & 0
\end{array}\right),\left(\begin{array}{cc}
* & * \\
* & *
\end{array}\right)\right), \quad V:=V_{0} \oplus V_{1} \oplus V_{2} .
$$

Then the 18-dimensional subspace $V \subset J_{s}$ is preserved by $G$. Each $V_{n}$ is an irreducible $\mathrm{SL}_{3} \rtimes \mathbb{Z} / 2$-representation. We could also write $V_{n}=\langle j, i j, k, i k, j k,(i j) k\rangle \subset \mathbb{O}$.

Theorem 3.1. $G$ acts on $V$ and $\mathbb{P}(V)$ generically freely.

Proof. For generic freeness, it is enough to assume the base field is algebraically closed. Let $p=(x, y, z) \in V_{0} \oplus V_{1} \oplus V_{2}$. To prove the theorem, we will first reduce to the case where $(x, y, z)$ is of a certain form, and then we will show the (scheme-theoretic) $G$ stabilizer of such a point is trivial. First we show that for a generic point $G_{p}(\bar{F})=\{1\}$, and then we show that $\operatorname{Lie}\left(G_{p}\right)=\{0\}$, which together imply $G_{p}=\{1\}([$ KMRT, 22.5] or $[\mathrm{BF} 03,4])$.

Notice the size of the $G$-stabilizer is unchanged along $G$-orbits. Generically we may assume the norm of $z$ (as an element of $\mathbb{O}$ ) is non-zero, so we can move it to a scalar multiple of $j \in V_{2}$ via an element of $\mathrm{SL}_{3} \subset G$. This is because $\mathrm{SL}_{3}$ acts transitively on norm one octonions orthogonal to $i$ and 1 [SV00, 2.2]. Next, we can find an $r_{\lambda} \in$ $T_{2} \subset G$ (which fixes $V_{2}$ ) that sends $y \in V_{1}$ to an element with zero $i j$ coefficient. Finally, $\mathrm{SL}_{2} \subset \mathrm{SL}_{3}$ acts transitively on norm one octonions in $\langle k, i k, j k,(i j) k\rangle$ [SV00, 2.2 , so we can assume $y$ is in the span of $j$ and $k$. In other words, we will assume $p=(x, y, z) \in V_{0} \oplus\langle j, k\rangle \oplus\langle j\rangle$.

Step 1: Prove $G_{p}(\bar{F})=\{1\}$. Assume $g \in G(\bar{F})$ fixes such an element. We need to show that for $(x, y, z)$ generic of this form, this implies $g$ is trivial. Firstly, notice that generically the norms of $x, y$, and $z$ are all distinct, and any non-trivial contribution from $S_{3} \subset G$ would permute the norms. So we may assume $g=\left(g_{0}, g_{1}, g_{2}\right) \in$ $\operatorname{Spin}_{8} \cap G \subset \mathrm{SO}(\mathbb{O})^{3}$.

The identity 1.4 together with $g_{2}(j)=j$ and $g_{0}(\langle 1, i\rangle)=\langle 1, i\rangle$ implies that $g_{1}$ preserves $\langle j, i j\rangle \subset V_{1}$. Due to our assumption on $y \in V_{1}$, which is fixed by $g_{1}$, we see that $g_{1}(j)=j$ and $g_{1}(k)=k$. So by the identity 1.4 we get $g_{0}(1)=1$.

Now let us write $g=f \cdot r_{\lambda} \cdot s_{\mu} \cdot \iota$, where $(f, \iota) \in \mathrm{SL}_{3} \rtimes \mathbb{Z} / 2$, and $s$ and $r$ are defined in 1.6. Notice that $g_{0}(1)=1$ implies that $\left(r_{\lambda} s_{\mu}\right)_{0}(1)=1$, which implies $\mu=\lambda^{-1}$.

Switching to the matrix perspective of the octonions (see 1.1), the identity $j=g_{1}(j)$ becomes $\left(\left(\begin{array}{ll}0 & 1 \\ 1 & 0\end{array}\right), 0\right)=\left(f \cdot r_{\lambda} \cdot s_{\lambda^{-1}}\right)_{1}\left(\left(\begin{array}{ll}0 & 1 \\ 1 & 0\end{array}\right), 0\right)=f \cdot\left(\left(\begin{array}{cc}0 & \lambda^{-1} \\ \lambda & 0\end{array}\right), 0\right)$. Since $f$ is an automorphism fixing the diagonal, this implies $\left.\left(\left(\begin{array}{cc}0 & 1 \\ -1 & 0\end{array}\right), 0\right)=f\left(\left(\begin{array}{cc}1 & 0 \\ 0 & -1\end{array}\right), 0\right) \cdot\left(\left(\begin{array}{cc}0 & \lambda^{-1} \\ \lambda & 0\end{array}\right), 0\right)\right)=$ $f\left(\left(\begin{array}{cc}0 & \lambda^{-1} \\ -\lambda & 0\end{array}\right), 0\right)$. Now using linearity of $f$, and a similar argument with $g_{1}(k)=k$, we 
get

$$
f\left(\left(\begin{array}{ll}
0 & a \\
b & 0
\end{array}\right), 0\right)=\left(\left(\begin{array}{cc}
0 & \lambda a \\
\lambda^{-1} b & 0
\end{array}\right), 0\right), \quad f\left(0,\left(\begin{array}{cc}
a & 0 \\
0 & b
\end{array}\right)\right)=\left(0,\left(\begin{array}{cc}
\lambda a & 0 \\
0 & \lambda^{-1} b
\end{array}\right)\right) .
$$

In $V_{0}$ we have that $\left(r_{\lambda} s_{\lambda^{-1}}\right)_{0}\left(\left(\begin{array}{cc}0 & x_{1} \\ y_{1} & 0\end{array}\right),\left(\begin{array}{cc}x_{2} & y_{3} \\ x_{3} & y_{2}\end{array}\right)\right)=\left(\left(\begin{array}{cc}0 & \lambda^{2} x_{1} \\ \lambda^{-2} y_{1} & 0\end{array}\right),\left(\begin{array}{cc}\lambda^{2} x_{2} & \lambda^{-2} y_{3} \\ \lambda^{2} x_{3} & \lambda^{-2} y_{2}\end{array}\right)\right)$, for any $x_{i}, y_{i} \in F$. There are now two cases to consider.

Case: $\iota=1$. In other words, $g_{0}(i)=i$. In this case, $g=f \cdot r_{\lambda} s_{\lambda^{-1}}$ acts in $V_{0}$ as $\left(\left(\begin{array}{ll}0 & a \\ b & 0\end{array}\right), 0\right) \mapsto\left(\left(\begin{array}{cc}0 & \lambda^{3} a \\ \lambda^{-3} b & 0\end{array}\right)\right.$, ). Since a generic $x \in V_{0}$ is fixed by $g$, this implies $\lambda^{3}=1$, which means $s_{\lambda^{-1}} r_{\lambda} \in \mathrm{SL}_{3}$, and therefore $g \in \mathrm{SL}_{3}$. But $g_{1}(k)=k$ and $g_{2}(j)=j$ imply $g$ also fixes $j$ and $k$, which means $g$ is trivial.

Case: $\iota \neq 1$. In other words, $g_{0}(i)=-i$. Let $x=\left(\left(\begin{array}{cc}0 & x_{1} \\ y_{1} & 0\end{array}\right),\left(\begin{array}{ll}x_{2} & y_{3} \\ x_{3} & y_{2}\end{array}\right)\right) \in V_{0}$. By the identity in 1.4 combined with $g_{1}(j)=j$ and $g_{1}(k)=k$ we have that $g_{0}(\langle j, i j\rangle)=\langle j, i j\rangle$ and $g_{0}(\langle k, k i\rangle)=\langle k, k i\rangle$. So we have

$$
\begin{aligned}
& g_{0}\left(\left(\begin{array}{cc}
0 & x_{1} \\
y_{1} & 0
\end{array}\right), 0\right)=\left(\left(\begin{array}{cc}
0 & \lambda^{3} y_{1} \\
\lambda^{3} x_{1} & 0
\end{array}\right), 0\right), \\
& g_{0}\left(0,\left(\begin{array}{cc}
x_{2} & 0 \\
0 & y_{2}
\end{array}\right)\right)=\left(0,\left(\begin{array}{cc}
\lambda^{3} y_{2} & 0 \\
0 & \lambda^{3} x_{2}
\end{array}\right)\right) .
\end{aligned}
$$

Since $x$ is generic and we are assuming it is fixed by $g_{0}$, this implies $\lambda^{3}=x_{1} / y_{1}$ and $\lambda^{3}=x_{2} / y_{2}$. But for generic $x$ these two ratios are not equal, which gives a contradiction, so no such $g$ exists. This proves that generically the stabilizer of $G$ on $V$ has trivial $\bar{F}$-points.

Step 2: Prove $G_{[p]}(\bar{F})=\{1\}$. Consider a generic element $[p]=[(x, y, z)] \in \mathbb{P}(V)(\bar{F})$; we must show no element of $G(\bar{F})$ sends $(x, y, z)$ to a scalar multiple of itself. Consider the $S_{3}$ action on the triple of norms up to scalar $[(n(x), n(y), n(z))]$, viewed as an element of $\mathbb{P}^{2}$. Since $S_{3}$ acts faithfully on $\mathbb{P}^{2}$, it acts generically freely there. If $g \in G(\bar{F})$ fixes $[(x, y, z)]$, this implies the $S_{3}$ contribution is trivial. So we can assume $(x, y, z)$ is sent to its negative.

As for Step 1, we may assume $(x, y, z) \in V_{0} \oplus\langle j, k\rangle \oplus\langle j\rangle$. So if $g=\left(g_{0}, g_{1}, g_{2}\right)$ sends it to its negative then $g_{2}(j)=-j, g_{1}(j)=-j$, and $g_{1}(k)=-k$. The identity 1.4 gives us that $g_{0}(j k)=j k$. Since $g$ preserves orthogonality in $\mathbb{O}$, this contradicts our assumption that a generic $x \in V_{0}$ is sent to its negative, so generically the stabilizer of $G$ on $\mathbb{P}(V)$ has trivial $\bar{F}$-points.

Step 3: Prove $\operatorname{Lie}\left(G_{p}\right)=\operatorname{Lie}\left(G_{[p]}\right)=\{0\}$. Notice that if $[p] \in \mathbb{P}(V)(\bar{F})$, then we have an inclusion of subgroups $G_{[p]} \subset \operatorname{Norm}_{G}(\langle p\rangle)$. In particular, it is enough to show that $\operatorname{Lie}\left(\operatorname{Norm}_{G}(\langle p\rangle)\right)=\{h \in \operatorname{Lie}(G) \mid h \cdot p \in\langle p\rangle\}=\{0\}$ for a generic $p \in V$.

So take $p \in V_{0} \oplus\langle j, k\rangle \oplus\langle j\rangle$ as above, and take $h \in \operatorname{Lie}(G)$ such that $h \cdot p \in\langle p\rangle$. Use the root space decomposition to write $h=h_{0}+\sum_{\alpha} h_{\alpha}$.

Here $(0,0, j)$ is supported on two weight spaces, $\pm(++--)$, see 1.7. One of these weight spaces lies in the standard $\mathrm{SL}_{3}$-representation inside $V_{2}$, while the other lies in its dual inside $V_{2}$. Lie $(G)$ preserves each $V_{n}$, so $h \cdot(0,0, j) \in\langle(0,0, j)\rangle$. If $\beta= \pm(++--)$, and $\alpha$ is an $\mathrm{SL}_{3}$-root such that $\beta+\alpha$ is a weight of $V$, then $h_{\alpha}=0$, because otherwise $h_{\alpha} V_{\beta}=V_{\alpha+\beta}$, which contradicts $h \cdot(0,0, j) \in\langle(0,0, j)\rangle$. So if $h_{\alpha} \neq 0$ then $\alpha= \pm\left(\epsilon_{3}-\epsilon_{4}\right)$. By arguing similarly with $\beta= \pm(+-++)$, or $\pm(++-+)$, we see $\alpha$ could not be $\pm\left(\epsilon_{3}-\epsilon_{4}\right)$ either. So $h \in \operatorname{Lie}\left(T_{4}\right)$. Therefore $h$ preserves the weight spaces.

Since we have assumed there exists a $\lambda \in F$ such that $h \cdot p=\lambda p$, considering the weights of $V_{0}$ (from 1.7), we see that $\epsilon_{2}(h)=\lambda$ and $-\epsilon_{2}(h)=\lambda$. Since char $F \neq 2$, we have $\lambda=0$. Looking at other weight spaces we see $\epsilon_{3}(h)=\epsilon_{4}(h)=0$ as well as $\epsilon_{1}(h)=0$. This implies $h=0$, and completes the proof that $G_{p}=G_{[p]}=\{1\}$. 
Proof of Theorem 0.3. By Theorem 2.6 we have that $\operatorname{ed}\left(F_{4}\right) \leq \operatorname{ed}(G)$ for the smooth 10-dimensional group $G$ defined above. By Theorem 3.1 we have a compression $V \rightarrow$ $\mathbb{P}(V)$ from a generically free $G$-representation to its 17 -dimensional generically free projectivization. Therefore $\operatorname{ed}\left(F_{4}\right) \leq \operatorname{ed}(G) \leq \operatorname{dim} \mathbb{P}(V)-\operatorname{dim} G=17-10=7$, as required (see also [BF03, Prop. 6.17]).

\section{REFERENCES}

[Alb47] A. A. Albert, A structure theory for Jordan algebras, Ann. of Math. (2) 48, (1947), 546-567.

[BF03] G. Berhuy, G. Favi, Essential dimension: a functorial point of view (after A. Merkurjev). Doc. Math. 8 (2003), 279-330.

[Bö11] C. Böhning, The rationality problem in invariant theory, preprint online.

[Bour] N. Bourbaki, Lie groups and Lie algebras. Chapters 46, Springer-Verlag (2002).

[Ga05] S. Garibaldi, Unramified cohomology of classifying varieties for exceptional simply connected groups, Transactions of the American Mathematical Society 358, No. 1 (2005) 359-371.

[Ga09] S. Garibaldi, Cohomological invariants: Exceptional groups and Spin groups, Memoirs of the American Mathematical Society, Number 937 (2009).

[GMS] S. Garibaldi, A. Merkurjev, J.-P. Serre, Cohomological invariants in Galois cohomology., University Lecture Series, 28. AMS (2003).

[Ja58] N. Jacobson, Composition algebras and their automorphisms, Rend. Circ. Mat. Palermo (2) 7 (1958) 55-80.

[Ja03] J.C. Jantzen, Representations of Algebraic Groups, Second Edition, Mathematical Surveys and Monographs, Vol 107, AMS (2003)

[KMRT] M.-A. Knus, A. Merkurjev, M. Rost, J.-P. Tignol, The book of involutions, Colloquium Publications Volume 44, American Mathematical Society (1998).

[Le04] N. Lemire, Essential dimension of algebraic groups and integral representations of Weyl groups, Transform. Groups 9, no. 4 (2004) 337-379.

[Mac08] M.L. MacDonald, Cohomological invariants of odd degree Jordan algebras, Math. Proc. Camb. Phil. Soc. 145 (2008) 295-303.

[Me13] A. Merkurjev, Essential dimension: A survey, Transform. Groups 18, no. 2 (2013) 415-481.

[Re00] Z. Reichstein, On the notion of essential dimension for algebraic groups, Transformation Groups 5 , no. 3 (2000) 265-304.

[Re10] Z. Reichstein, Essential dimension, ICM proceedings, (2010).

[Se93] J.-P. Serre, Cohomologie galoisienne: progrès et problèmes, Séminaire N. Bourbaki, no. 783 (1993) 229-257.

[SV00] T.A. Springer, F.D. Veldkamp, Octonions, Jordan algebras and exceptional groups. Springer (2000). 